\title{
Validation of the D'Alembert's Equation for the Vibrating String Problem
}

H. LECKAR ${ }^{1}$, Instituto de Matemática, Universidade Federal Fluminense (UFF), Rua Mario Santos Braga s/n, 24020-140 Niterói, RJ, Brasil

R. SAMPAIO ${ }^{2}$, Depto de Engenharia Mecânica, Pontifícia Universidade Católica do Rio de Janeiro (PUC-Rio), Rua Marquês de São Vicente 225, 22453-900 Gávea, RJ, Brasil

E. CATALDO ${ }^{3}$, Depto de Matemática Aplicada, Universidade Federal Fluminense (UFF), Programa de pós-graduação em Engenharia de Telecomunicações (UFF), Programa de pós-graduação em Engenharia Mecânica (UFF), Rua Mario Santos Braga s/n, 24020-140 Niterói, RJ, Brasil.

\begin{abstract}
The aim of this work is to discuss the dynamics of an one spacedimension structure without flexural stiffness. We model the behavior of a very light string and we present the problem, considering large deformations, considering that each particle has only vertical motion. We discuss the kind of constitutive equations needed in such motion. Using a perturbation method, we show that the D'Alembert's equation can be seen as a first approximation for a general model and, then, its limitations become clear.
\end{abstract}

\section{Introduction}

The dynamics of an one space-dimension structure, a string, can be modelled considering several levels of complexity. The simplest one makes a strong geometrical hypothesis: The string moves in a plane and each one of its points has only vertical displacement. This hypothesis simplifies the problem and it allows us to represent the position of each point, at a instant $t$, by a continuous real function.

This hypothesis produces good models, that is, models capable of good predictions from the Engineering point of view. However, some problems can not be described by this model. For instance, the case of an inextensible string with fixed extremities. We will show that this case must be governed by a special constitutive equation. But, if we introduce a parameter and if we use some perturbation techniques, we can achieve a good approximation for the problem.

\footnotetext{
${ }^{1}$ http://www.professores.uff.br/hfleckar

${ }^{2}$ http://www.mec.puc-rio.br/prof/rsampaio/rsampaio.html

${ }^{3}$ http://www.professores.uff.br/ecataldo
} 
The second level of complexity considers the string as a curve in space. This model is a better representation of the reality. While the first model considers an inextensible string, that is, a string that in all motions preserves the length of any of its parts, the other one considers an elastic string. In this case, to complete the model, we have to show how the forces in the string are related to deformation.

The third level, and more complete, uses a Cosserat Continuum model. In this case, for each point of the string, we associate an orthogonal basis that describes the string cross section behavior. With this basis, the string can be torsioned, for example. This case, however, will not be discussed here.

Generally, one introduces the wave motion and the normal mode analysis of a one-dimensional continuum considering small oscillations in an ideal elastic string. With appropriate assumptions, a linear wave equation is readily derived and its analysis is a guide to the study of the wave propagation problem in other systems of relevance. However, such idealization may not offer any guidance for situations where disturbances are not necessarily small compared with the dimensions of the system. In large-amplitude oscillations of a real string, the longitudinal and transverse motions become coupled and a realistic treatment relies on a careful consideration of the appropriate constitutive relations linking the configuration of the system to the elastic forces exerted by the medium. Such considerations become importants if one wants to distinguish the behaviour of a rubber band from that of a violin string. The constitutive relations for such realistic systems, generally, rise to a non-linear wave equation. There is also a class of problems where the elastic forces are provided by the constraint of inextensibility. Such problems clearly lie outside the scope of treatments rooted in the use of linear elastic laws.

\section{Classical Modelling of a Vibrating String, Con- sidering Small Displacements}

\subsection{Standard derivation of the vibrating string equation}

Let us consider the simplified problem of a vibrating string. We represent the position of a point, at a instant $t$, by a continuous real function $u:[0, L] \times[0, \infty) \rightarrow$ $\mathbb{R},(x, t) \rightarrow u(x, t)$, where $[0, L]$ represents the string in the reference frame. At this point, we make an important hypothesis; taking into account the hypothesis inherent to the linear problem:

Hypothesis: In a string there are only traction efforts.

Let $T_{x}$ be the force exerced by the part of the string on the left side of $x$ into the part on the right side of $x$.

Let us make a forces balance in the segment $[x, x+\Delta x]$ ( $u$ direction):

$$
\overbrace{(\rho \Delta x)}^{\text {mass }} \frac{\partial^{2} u}{\partial t^{2}}=T_{x+\Delta x} \operatorname{sen} \theta_{x+\Delta x}-T_{x} \operatorname{sen} \theta_{x}+\int_{x}^{x+\Delta x} f(s) d s .
$$

Supposing that there are no external forces and no inertia in the $x$ direction, we have: 


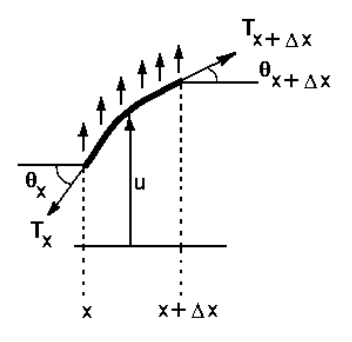

$$
T_{x+\Delta x} \cos \theta_{x+\Delta x}=T_{x} \cos \theta_{x} .
$$

By considering $\theta_{y}$ small for any $y$, we have:

$$
T_{x+\Delta x}=T_{x},
$$

because

$$
\cos \theta_{x+\Delta x} \cong \cos \theta_{x} \cong 1,
$$

and the traction $T$ in the string is constant, independent on $x$. Using

$$
\sin \theta_{y} \sim \operatorname{tg} \theta_{y}=\left.\frac{\partial u}{\partial x}\right|_{y}
$$

we have

$$
\rho \Delta x \frac{\partial^{2} u}{\partial t^{2}}=\left.T \frac{\partial u}{\partial x}\right|_{x+\Delta x}-\left.T \frac{\partial u}{\partial x}\right|_{x}+f(s) \Delta x .
$$

Dividing by $\Delta x$ and taking $\Delta x \rightarrow 0$, we have:

$$
\rho \frac{\partial^{2} u}{\partial t^{2}}=\frac{\partial}{\partial x}\left(T \frac{\partial u}{\partial x}\right)+f
$$

that is,

$$
\rho \frac{\partial^{2} u}{\partial t^{2}}=T \frac{\partial^{2} u}{\partial x^{2}}+f
$$

\section{Observations:}

1) in technical language, a string is a structural element that do not resist to compression efforts. When compressed, by loads applied in its extremities, the string reacts changing its shape. In the study of a string transverse motions, it is not necessary any supplementary hypothesis. We must observe that the expression $\frac{\partial u}{\partial x}$ is the inclination of the string, at a point $x$, in the instat $t$, and not the deformation, as appears when we study longitudinal waves propagation along a beam. 
2) if the string is in a configuration such that the distribution of forces in the longitudinal direction, $g$, is important [we say the reference configuration is vertical and we want to consider the weight], then:

$$
T_{x+\Delta x} \cos \theta_{x+\Delta x}-T_{x} \cos \theta_{x}=\int_{x}^{x+\Delta x} g(s) d s .
$$

For small $\theta_{y}$ we have:

$$
T_{x+\Delta x}-T_{x}=\int_{x}^{x+\Delta x} g(s) d s
$$

that implies,

$$
\frac{d T}{d x}=g
$$

In this case $T$ is not constant.

\subsection{A flexible string with correction of the tension term}

In the following, we will use subscripts, in each corresponding variable of derivation, to denote partial derivatives.

The equation of the vibrating string motion is valid only for large pre-stressed tension, $T$, and small values of the string element gradient, $u_{x}$. In the practical case of a finite string, the solution takes on a standing wave form set by the boundary conditions. The impact of a particle with a flexible string theory cannot rely on the string elastic properties; that is, it is considered that the tension does not increase as the string is stretched. The basic problem is how to devise a strategy which will describe the changing of the string shape and its velocity profile during the impact. In the vibrating string equation, the string tension was considered much greater than any increasing caused by stretching. So, the string element length $\delta s$ was the same as $\delta x$. A modified equation, which takes account of finite stretching, could be:

$$
\rho u_{t t}=[T+Y(d s / d x-1)] u_{x x} .
$$

Knowing that $d s=\left[1+u_{x}^{2}\right]^{1 / 2} d x$ and neglecting the third and higher powers, one has:

$$
u_{t t}=\left[c_{1}^{2}+(1 / 2) c_{2}^{2} u_{x}^{2}\right] u_{x x},
$$

where $c_{1}^{2}=T / \rho$ is the speed of transverse waves along the string, $c_{2}^{2}=Y / \rho$ is the speed of compressional longitudinal waves in the string and $Y$ is the Young modulus.

This model introduces a correction in the string tension. But, it can only describe small motions due to the hypothesis we made for the geometry.

\subsection{A simple problem for a string}

Let us now discuss the validity of subsections 2.1 and 2.2 . 
Let us consider the problem of a string where no assumption of small motion is done, but we say that a particle moves only in a vertical direction in a plane.

A string of initial length $L$ is fixed at both ends. We search a constitutive equation such that a plane motion of the type

$$
(s, t) \longrightarrow(x(s, t), y(s, t))=(s, u(s, t)), \mathbf{r}(s, t)=(s, u(s, t))
$$

would be possible.

It means that a point $s$ moves only in the vertical direction. Of course such string cannot be inextensible. It must change its length in order to have the motion prescribed.

The motion equation is:

$$
\rho \mathbf{r}_{t t}=T_{s} \mathbf{r}_{s}+T \mathbf{r}_{s s}+f
$$

Let us take $f=0$ and, by hypothesis:

$$
\begin{gathered}
\mathbf{r}(s, t)=(s, u(s, t)) \Rightarrow \mathbf{r}_{t t}(s, t)=\left(0, u_{t t}(s, t)\right), \mathbf{r}_{s}(s, t)=\left(1, u_{s}(s, t)\right), \mathbf{r}_{s s}=\left(0, u_{s s}(s, t)\right) ; \\
T_{s}\left(1, u_{s}\right)+T\left(0, u_{s s}\right)=\rho\left(0, u_{t t}\right),
\end{gathered}
$$

then, $T_{s}=0$, that is, $T$ is constant in space, depending only on time:

$$
\rho u_{t t}=T(t) u_{s s} .
$$

Observe that neither $\rho$ nor $T$ needs to be constant.

There is, at least, one constitutive equation that is according to this result.

Suppose $S(t)$ the length of the string at time $t$,. Then, $S(t)=\int_{0}^{L} \sqrt{1+u_{s}^{2}} d s$.

Obviously $S(t)-L \geq 0$ and a possible constitutive equation is:

$$
T(t)=T_{0}+k\left(\frac{S(t)-L}{L}\right) .
$$

This equation is an odd one. The tension changes only with the total length of the string; a rubber band does not have this behavior, for example.

Since $\left|\mathbf{r}_{s}\right|=\sqrt{1+u_{s}^{2}}$ may depend on $s$ a local constitutive equation would not be allowable, and if $\left|\mathbf{r}_{s}\right|$ is independent on $s$ means that if $\mathbf{r}_{s}$ is constant then $u$ is linear in $s$ and since $u(0, t)=0, u(L, t)=0$, the only possible motion would be $u=0$.

Now let us consider one extreme fixed and the other with prescribed motion, say $L(t)=L(1+v t), v$ fixed.

Clearly, a solution with the form $(s, u(s, t))$ has no meaning, so, we search a solution as $\mathbf{r}(s, t)=(s(1+v t), u(s(1+v t), t))$.

We have:

$$
\begin{aligned}
& \mathbf{r}_{t}=\left(s v, u_{t}+u_{s}(s v)\right) \\
& \mathbf{r}_{t t}=\left(0, u_{t t}+u_{t s}(s v)+u_{s t}(s v)+u_{s s}(s v)^{2}\right), \\
& \mathbf{r}_{s}=\left(1+v t, u_{s}(1+v t)\right), \\
& \mathbf{r}_{s s}=\left(0, u_{s s}(1+v t)^{2}\right)
\end{aligned}
$$


then,

$$
\rho\left(0, u_{t t}+2 u_{s t}(s v)+u_{s s}(s v)^{2}\right)=T_{s}\left(1+v t, u_{s}(1+v t)\right)+T\left(0, u_{s s}(1+v t)^{2}\right),
$$

that implies $T_{s}=0$ and

$$
\rho\left[u_{t t}+2 u_{s t}(s v)+u_{s s}(s v)^{2}\right]=T u_{s s}(1+v t)^{2} .
$$

\section{Dynamics of Strings: Inextensible Model, Elas- tic String and Perturbation Techniques}

Intuitively, a string is a perfectly flexible structural element, that is, unable to support any flexural efforts. In a string, the efforts always act in the tangent direction. This hypothesis simplifies the problem because, known the configurations, the tension is completely determined by a scalar field.

Usually, the motion equation of a string is presented directly as a linear equation. But this brings some conceptual difficulties, as we will show. Here, we have chosen to present an exact model, valid for small and large motions. Only after having discussed the physics of the problem, we linearize the equation to compare it with the classic model presented in section 2 . Some boundary conditions are lost in this process and, depending on the hypothesis adopted, we may have more than one linearization. It is not a surprise! We will discuss two different models of strings: one considers the string inextensible and other considers it elastic.

We described the string motion by a continuous vector function:

$$
\begin{aligned}
& \mathbf{r}:[0, L] \times[0,+\infty) \rightarrow \mathbf{R}^{3} \\
& (s, t) \rightarrow \mathbf{r}(s, t)
\end{aligned}
$$

The variable $s$ denotes a material point of the string, $L$ is its total length, $\mathbf{r}(s, t)$ is position vector of the string for the point $s$, at instant $t ; \mathbf{r}(\cdot, t)$ describes a configuration at the instant $t$ and $\mathbf{r}(s, \cdot)$ is the trajectory of the point $s$.

The interval $[0, L]$ can be thought as the reference (or natural) frame of the string, that is, a frame in the which the resultants force and moments on any part of the string is zero.

\subsection{A model for an inextensible string}

The name inextensible string, that is, arc-length constant for all $t$, is justified by the equation $\int_{a}^{b} \mathbf{r}_{s} \cdot \mathbf{r}_{s} d s=b-a$, for all $a, b$ in $[0, L]$ and all $t>0$, where the dot represents the inner product. This is equivalent to the restriction:

$$
\mathbf{r}_{s} \cdot \mathbf{r}_{s}=1, \forall t>0
$$

The equation (3.1) expresses the fact that the length of any part of the string stays unchanged during the motion. 
The equation of the motion of the string can be achieved by means of a forces balance; we will deduce this equation using the Hamilton's principle.

Let us build the system's Lagrangean. We take $(1 / 2) \rho \mathbf{r}_{t} \cdot \mathbf{r}_{t}$ as the kinetic energy density and $\mathbf{f} \cdot \mathbf{r}$, is the energy density due to an external force, $\mathbf{f}$, where $\rho$ is the mass linear density per unit-length of the string. The term of elastic potential energy does not appear due to the inextensibility of the string (there is no mecanism to accumulate energy) and, finally, the restriction (3.1) is taken into account by means of Lagrange's multipliers. In that case, due to the nature of the restriction (3.1), the multiplier is a function of $(s, t)$.

The Lagrangean, in that case, is:

$$
\mathcal{L}\left(t, \mathbf{r}, T, \mathbf{r}_{t}, \mathbf{r}_{s}\right)=\int_{0}^{T}\left[(1 / 2) \rho \mathbf{r}_{t} \cdot \mathbf{r}_{t}-\mathbf{f} \cdot \mathbf{r}-(1 / 2) T\left(\mathbf{r}_{s} \cdot \mathbf{r}_{s}-1\right)\right] d t .
$$

We chose the Lagrange's multiplier in the form $-T / 2$. The Euler-Lagrange's equations of $\mathcal{L}$ is:

$$
\rho \mathbf{r}_{t t}=\left(T \mathbf{r}_{s}\right)_{s}+\mathbf{f}
$$

with the restriction (3.1).

Equation (3.2) describes the motions of the system and we see immediately that $T$ can be interpreted as the tension in the string, a scalar field, and $T \mathbf{r}_{s}$, a vector field, as the force that one part of the string exerces on the other contiguous part.

Differentiating (3.1) we obtain two equations whose physical interpretation should be obvious to the reader, $\mathbf{r}_{s} \cdot \mathbf{r}_{s s}=0, \quad \mathbf{r}_{s} \cdot \mathbf{r}_{s t}=0$ and, differentiating (3.2) and taking the inner product with $\mathbf{r}_{s}$, then

$$
T_{s}=\left(\rho \mathbf{r}_{t t}-\mathbf{f}\right) \cdot \mathbf{r}_{s}
$$

We see that the gradient of tension is equal to the tangencial component of the force of inertia minus the external force. In the following, for simplicity, and because the extension of these results is obvious, we will consider $\mathbf{f}=0$.

Equations (3.1) and (3.2), together with initial and boundary conditions, completely characterize the dynamics of the string.

Now, we have to establish a mathematical problem. We have to give initial and boundary conditions to solve (3.1) and (3.2), and it is not simple. Considering only plane motions, we may write $\mathbf{r}=(x, y, 0)$ and the equations (3.1) and (3.2) can be written in terms of functions $x, y$ and $T$, which is to be determined in the domain $[0, L] \times[0,+\infty)$. That problem is highly non-linear and, of course, one needs an theorem of existence to show that the problem has a meaningful.

How many initial conditions should be imposed to have an unique solution to the initial-boundary value problem? The new equations for $x$ an $y$ are of second order in $t$, then the answer seems to be four conditions, two for $x$ and two for $y$. On the other hand, equation (3.1) relates $x$ to $y$, what seems to indicate that only two conditions are needed. See [5] for details. 


\subsubsection{Approximation for small oscillations}

Now, we will consider the string motion in a way that its configurations are always close of the $x$ axis. More precisely, the coordinate $y(s, t)$ has order of magnitude $\varepsilon<<1$ and we can write

$$
y(s, t)=\varepsilon y_{0}(s, t),
$$

where we does not make restriction for $y_{0}$. Our parameter of disturbance will be $\varepsilon$.

As $y_{s}=0(\varepsilon),(3.1)$ implies that $x_{s}=1+0\left(\varepsilon^{2}\right)$ and we obtain by integration:

$$
x(s, t)=s+x_{0}(t)+\varepsilon^{2} x_{1}(s, t) .
$$

Now, without more discussion, we will make the following hypothesis:

$$
x_{0}(t)=0 .
$$

From the equation (3.2) we conclude that:

$$
T(s, t)=T_{0}(s, t)+\varepsilon^{2} T_{1}(s, t) .
$$

The fact of (3.3) only have odd powers of $\varepsilon$, while (3.4) and (3.5) only have even powers, it is consonant with our physical expectation. To change $\varepsilon$ by $-\varepsilon$ corresponds to take configurations that are images in relation to the $x$ axis, and then the tension should not vary.

We will find the equations solutions for $x$ and $y$ until the $\varepsilon^{2}$ order. We substitute $(3.3),(3.4)$ and (3.6) in the corresponding equations. By taking into account that $\varepsilon<<1$, we have:

$$
\begin{gathered}
\text { approximation order } \varepsilon^{0}: \frac{\partial T_{0}}{\partial s}=0 \\
\text { approximation of } \varepsilon^{1} \text { order }: \rho \frac{\partial^{2} y_{0}}{\partial t^{2}}=\frac{\partial}{\partial s}\left(T_{0} \frac{\partial y_{0}}{\partial s}\right) \\
\text { approximations of } \varepsilon^{2} \text { order }: 2 \frac{\partial x_{1}}{\partial s}+\left(\frac{\partial y_{0}}{\partial s}\right)^{2}=0 ; \\
\qquad \frac{\partial^{2} x_{1}}{\partial t^{2}}=\frac{\partial T_{1}}{\partial s}+\frac{\partial}{\partial s}\left(T_{0} \frac{\partial x_{1}}{\partial s}\right)
\end{gathered}
$$

Equation (3.7) implies $T_{0}(s, t)=T_{0}(t)$, that is: the smaller order term of the tension is spatially constant . The magnitude of $T_{0}$ is specified through boundary conditions. We will make the hypothesis that $T_{0}$ is constant. This hypothesis is not vital but it simplifies greatly the problem. To find the approximation up to $\varepsilon^{2}$ order, we should initially to solve (3.8) and calculate $y_{0}$. Next, we solve (3.9), we evaluate $x_{1}$ and finally, we solve (3.10) and found $T_{1}$. The initial and boundary conditions needed to integrate (3.8)-(3.10) are well-known. 


\subsection{Elastic or extensible string}

In the previous section we studied some consequences of the inextensibility hypothesis. One of them is that the tension is not constitutive, that is, the tension does not depend on the material that composes the string. The tension comes from the inextensibility and, in the motion, the required tension to maintain the constant length of any part of the string should be produced. It is of the same nature as the pressure in the case of incompressible fluids.

In this section, we relax the inextensibility hypothesis, but we maintain the hypothesis of the string to be perfectly flexible. This hypothesis lead us to a problem: now, the length of the string is variable. In this case, the domain of arch length function $s$ is variable, being more convenient to take as independent variable, not $s$, but $m$, the string mass, measured from a fixed initial point $O$ in the string. Then, we take, as independent variable, the specific length $\nu=d s / d m$ of the string, instead of the specific mass $\rho=d m / d s$.

Let $\mathbf{r}(m, t)$ be the vector position of a point $m$ in the string at the instant $t$ and $E(\nu)$ the elastic potential energy, by unit of mass, accumulated by the string. We do not consider external forces, to simplify the problem. This assumption do not cause troubles, only more complex formulas. We suppose that the elastic potential energy dependence on $m$ is not explicit, what implies the "homogeneity of the material" which the string is made. There is not also any problem in considering the most general case $E(m, \nu)$, or even $E(m, t, \nu)$; case in which the material of the string is time varying.

The system's Lagrangean is:

$$
\mathcal{L}\left(t, \mathbf{r}, \mathbf{r}_{t}, \mathbf{r}_{m}\right)=\int_{0}^{M}\left[(1 / 2) \mathbf{r}_{t} \cdot \mathbf{r}_{t}-E(\nu)\right] d m,
$$

where $M$ it is the total mass of the string.

Because $s$ is the arch length, we have:

$$
\mathbf{r}_{m} \cdot \mathbf{r}_{m}=\nu^{2}
$$

By using this formula, the Euler-Lagrange's equation of (3.11) is,

$$
\mathbf{r}_{t t}=\left((1 / \nu) E_{\nu} \mathbf{r}_{m}\right)_{m} .
$$

Observe that $\mathbf{r}_{m} / \nu$ is equal to $\mathbf{r}_{s}$ and $E_{\nu}=T$ is the tension in the string, being $T$ a function of only one variable $\nu$, to compare with the results of the section 3.1. .

Equation (3.13) is a system of non-linear hyperbolic equations when hypothesis are made about the behavior of $E$. For simplifying, we consider plane motions, that is, $\mathbf{r}=(x, y, 0)$.

By analogous considerations made in the section 3.1., we will try to find a solution in the form:

$$
\begin{aligned}
& x(t, m)=\nu_{0}\left(m+\varepsilon x_{1}(m, t)+\varepsilon^{2} x_{2}(m, t)\right), \\
& y(t, m)=\nu_{0}\left(m+\varepsilon y_{1}(m, t)+\varepsilon^{2} y_{2}(m, t)\right) .
\end{aligned}
$$


Using the equations (3.12) and (3.13), we achieve, after calculations

first order approximation:

$$
\frac{\partial^{2} x_{1}}{\partial t^{2}}=c^{2} \frac{\partial^{2} x_{1}}{\partial m^{2}}, \quad \frac{\partial^{2} y_{1}}{\partial t^{2}}=c^{2} \frac{\partial^{2} y_{1}}{\partial m^{2}}
$$

These equations are natural waves of first order.

Resumo. Este trabalho tem como objetivo tratar a dinâmica de uma estrutura unidimensional que não tem qualquer rigidez a flexão. Ela modela o comportamento de uma corda muito fina, por exemplo. Discute-se um problema de grandes deformações no qual cada partícula tem um movimento puramente vertical e o tipo de equação constitutiva é necessária para possibilitar tal movimento. Usando um esquema de perturbação mostra-se que a equação de D'Alembert pode ser interpretada como uma primeira aproximação para um modelo geral e suas limitações se tornam evidentes.

\section{References}

[1] S.S. Antman, The equations for large vibrations of string, American Mathematical Monthly, 87 (1980), 359 - 370.

[2] S.S. Antman, "Non-Linear Problems in Elasticity", Springer, Applied Mathematical Sciences 107 , Berlin, 1991.

[3] J.E. Bolwell, The flexible string's neglected term, Journal of Sound and Vibrations, 206 (1997), 618-623.

[4] J.B. Keller, Large amplitude motion of a string, American Journal of Physics, 27 (1959), 584-586.

[5] H.F. Leckar and R. Sampaio, "Mathematical Aspects of Mechanical Vibrations", Notes in portuguese, 49 Brazilian Seminar of Analysis, UNICAMP, Campinas, 1999.

[6] R. Sampaio, "Topics in Wave Propagation", Notes in Portuguese, III School of Applied Mathematics, LNCC- CNPq, Rio de Janeiro, 1982. 